\title{
Impacted maxillary canines: tunnelised or open traction:
}

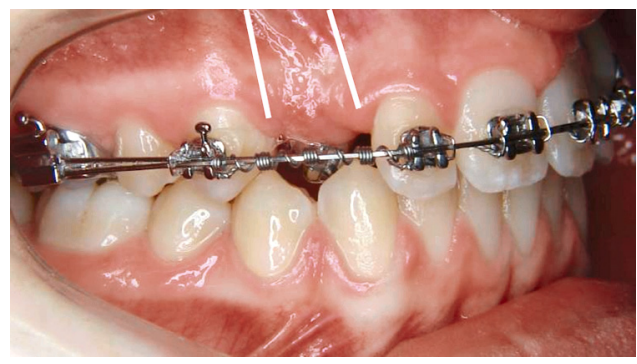

\author{
Herman VAN BEEK
}

\section{SUMMARY}

Impacted upper canines occur, in buccal or palatal sites, in about 3\% of the population and pose a threat to neighbouring teeth. Before orthodontists can bring them into their proper positions in the arch, oral surgeons often have to uncover them. In this article we discuss the traction pathways, open or tunnelised, that orthodontists can then use to begin moving them. While orthodontists can use both techniques successfully, they should understand the advantages and disadvantages of both before making a decision.

\section{KEYWORDS}

Canine

Impaction

Open traction

Tunnelised traction. 


\section{1 - INTRODUCTION}

Impacted upper canines occur in about $3 \%$ of the population and pose a threat to neighbouring teeth that sometimes undergo rapid and serious resorption. The palatal impaction of upper canine teeth may result from an excess of space, rather than lack of space, which is the cause of buccal impaction. Trauma is another possible cause of canine impactions and, cer-

\section{2 - SURGICAL PROCEDURES}

\section{- Labially impacted}

\section{canines}

These teeth can be uncovered even when they lie in a high position but in the process the attached gingiva will be disturbed making it advisable for the oral surgeon to attach a gingival graft at the cemento-enamel junction to prevent continuing recession. This flap will move with the canine as the orthodontist draws it into place ${ }^{3}$.

At the beginning of closed or tunnelised traction, after laying the flap, the surgeon bonds an attachment to the crown of the uncovered tooth. The surgeon then repositions the flap so that a ligature or gold chain can hang down from the tooth to an area where it is accessible ${ }^{4}$.

\section{- Palatally impacted}

\section{canines}

Two methods are available to orthodontists in treating these teeth.

In the first method, open traction, the oral surgeon exposes the impacted tooth and removes any bone that may impede its movement, the oral cavity and places a dressing in this tainly a genetic component also plays a role in the etiology. Timely removal of the deciduous canine in 10 or11 year-old children can sometimes prevents this problem from developing, but often surgical intervention may be required to retrieve over-retained canine teeth and prevent them from damaging neighbouring teeth.

space to keep it open for the tooth. Spontaneous eruption of the impacted

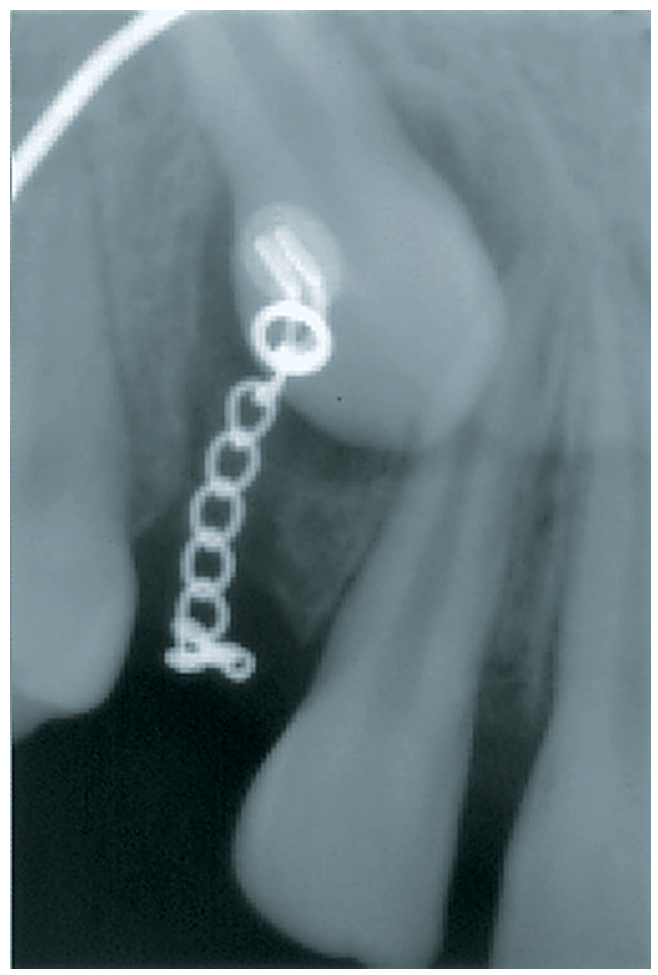

Figure 1

A radiograph showing a chain one end of which is attached to a button bonded to the canine's uncovered crown with the other end hanging into the oral cavity. 
tooth may follow this procedure even if the canine had occupied a particularly unfavourable position.

In the second method the surgeon uncovers the tooth, lays back a flap, removes the bone covering the crown of the exposed tooth, and bonds an attachment with a ligature or a gold chain is affixed to the crown. The surgeon then repositions the flap on the tooth with the ligature or chain passing through it in the direction along which the orthodontist will move the tooth (fig. 2).

Both of these methods, correctly applied, enjoy high rates of success, even though the controversy between the advocates of the two remains spirited.

Schmidt and Kokich ${ }^{2}$ prefer encouraging spontaneous eruption, arguing that by delaying bonding an attachment to the crown they give the tooth added time to begin erupting on its own initiative and that after this happens the orthodontist will have improved conditions, primarily of access and absence of bleeding and seepage, to bond correctly.

In addition, after a delay, the orthodontist will find it easier to inspect the tooth's progress and to plan the application of force to it. With the tunnel method, if the attachment becomes unbonded the surgeon and orthodontist will not know if enough bone had been removed or if the tooth is moving along the desired path and another surgical procedure may be indicated.

In the case of a canine frankly erupted into the palate, the oral surgeon lays back a flap and removes all the bone that might prevent the tooth from beginning to erupt and takes the precaution of suturing the

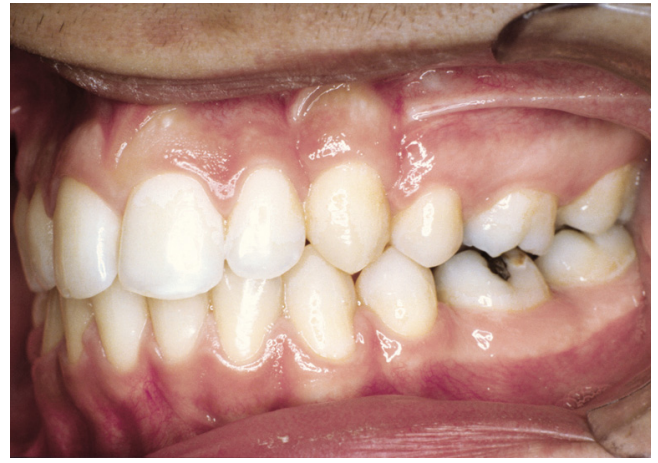

Figure 2

Intraoral photograph showing a formerly impacted upper left canine that had been brought into place with orthodontic traction. The graft of a flap repositioned apically has followed the tooth into its new position and can be seen as a thickened collar of attached gingiva.

palatal artery to prevent any blood from flowing. The surgeon cuts away part of the flap and leaves an opening over the tooth's crown that is filled with a surgical dressing. The surgeon removes the dressing a week later and asks the patient's parents to keep

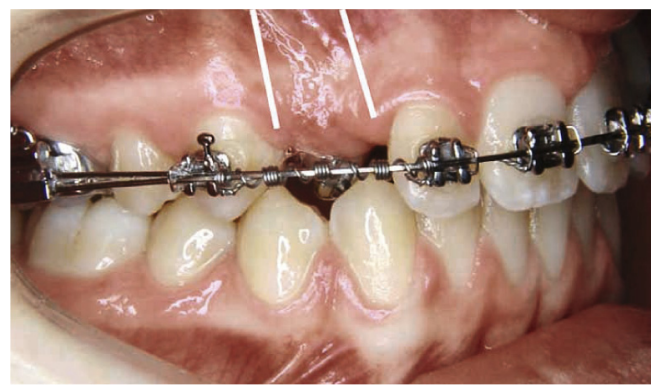

Figure 3

The oral surgeon has incised a buccal flap in order make two osteotomy cuts with a fine round bur in the cortical bone one mesial and one distal to the canine as show by the white lines in photo. 
the healing area clean and to watch possible growth of exuberant tissue that would fill the opening. Usually the canine then erupts spontaneously into the palate.

As soon as possible, the orthodontist should bond a bracket to the crown's labial surface and begin to move it slowly into place using light forces. After several months movement will grow slower because of the growth of scar tissue around the bracket and, in general, because cortical bone will lie in the planned path of tooth movement.

This is the time for a second intervention by the oral surgeon who

\section{3 - DISCUSSION}

The two methods, open and tunnelised traction, have been analysed scientifically and the results for both have been shown to be good and comparable to each other.

It would seem to us that opting for an open approach would be the logical choice because it allows the orthodontist to delay bonding a bracket to the uncovered canine to a time when conditions for this procedure have improved.

But it must be remembered that it requires two surgical interventions, even though the second one is considerably less painful for the patient, who, of course in accordance with the principles of informed consent, must be aware of its necessity from the outset of treatment.

In addition, there is more vertical relapse with the orthodontic movement of a buccally impacted canine into place with the open method than there is with the tunnelised technique. will lay a buccal flap and with a fine round bur will make cuts mesial and distal to the canine for a vertical osteotomy (fig. 3). The surgeon lays the flap back and adjusts its gingival contour. The canine will then progress rapidly to a high position where the orthodontist leaves it for a 3 month period as the palatal bone consolidates to form a point of resistance against which the canine will tip when the orthodontist pulls it down towards its final position. This method creates the typical bulge about the canine root without the orthodontist having had to apply buccal root torque to the tooth.

And, finally, the pigmentation of the displaced flap of attached gingiva may turn out to be aesthetically unacceptable.

But the tunnelised method also has a major disadvantage. If the oral surgeon has not removed enough bone, the impacted tooth's initial movement will be slow because its follicular sac which possessed the potential for bone resorption has been removed, although Becker ${ }^{1}$ and his associates do not agree with this conclusion.

And when the gold chain to which the orthodontic traction is applied is incorrectly oriented, its activation may be painful for the patient. In addition the orthodontist, working blind in the tunnelised method, cannot observe the tooth's progress or the vectors of force and accurately judge the times when it is necessary to adjust them.

The bracket may also have been placed in an awkward position. Even 


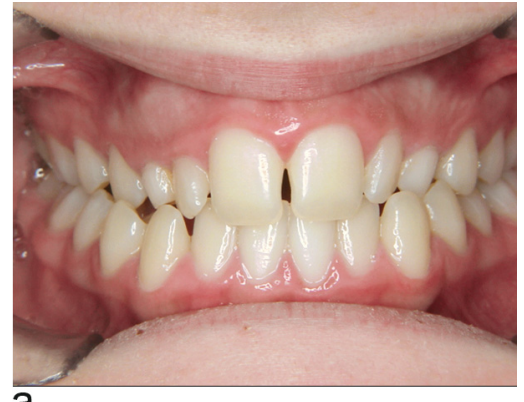

a

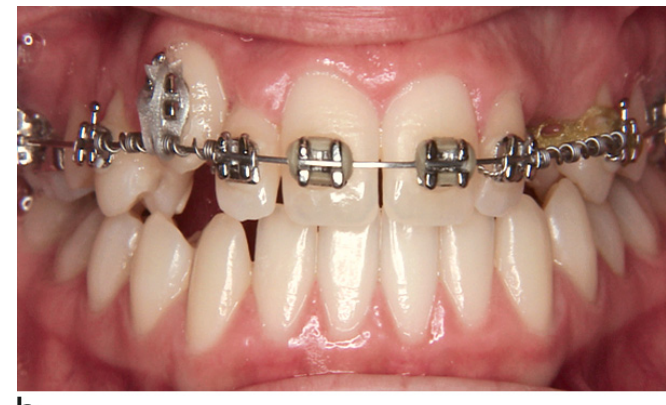

b

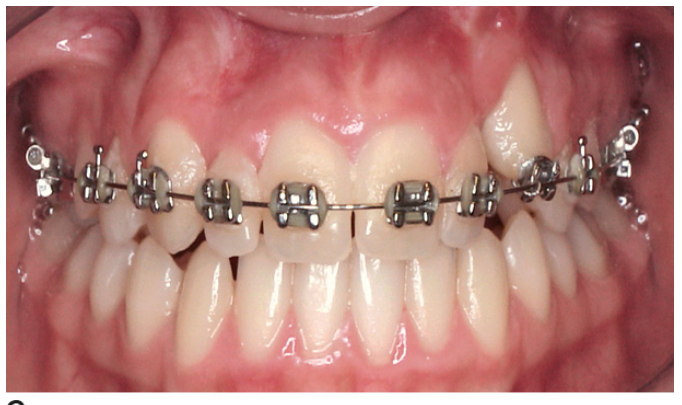

C

Figures 4 a to $c$

Intraoral photographs of a case where both maxillary canines were impacted, the right buccally, the left palatally (a). In photograph $b$, the right canine has erupted and is being pulled down into position. The left palatally impacted canine has been uncovered but is not visible. In view $c$ the left canine that had initially been moved horizontally in a high position toward its buccal position is now being drawn slowly occlusally with force applied to a bracket bonded to the tip of its cusp. Treating impacted canines in this way orthodontist can move them into the arch in correct axial inclination needing no torque adjustment.

so, if the orthodontist applies force vectors properly, there will be no problem. But the possibilities for error are greater in the closed, tunnelized technique and it is more complicated and more difficult to control and to execute.

It would improve the mechanics of treatment if the orthodontist could place the bracket on both the tooth's buccal surface and the tip of its cusp, but this cannot be done at the same time. It is also important for orthodontists to keep the canine in high positions to spare them from the forces of occlusion and to keep them there as they are moved horizontally above their future arch position. Then, after a rest period, the orthodontist can begin to move them down toward the occlusal plane while at the same correcting any incorrect axial root inclinations (fig. 4 a to c). It is a serious mistake to first move the canine down into position with a spring arm and next try to move it buccally because at this level the risk of interference from the mandibular canine is great. In addition if the crown is already in place it will take a long time for the orthodontist to torque its root buccally.

Forced eruption is no longer considered to be a good option. If the tooth erupts spontaneously the possibility of its becoming ankylosed is extremely slim. But by forcing its eruption, the orthodontist puts it into an over-corrected position, which implies that strong torque forces will have to be applied, prolonging treatment time and increasing the risk of resorption.

The conclusion that orthodontists should move palatally impacted canine teeth horizontally toward their final buccal destination while they remain in a high position derives from the observation of many authors that ectopically placed canines require palatal root torque when they are brought down too quickly toward the occlusal plane. But orthodontists can utilize this tendency for the prominence of the canine root to increase to 
their advantage if they follow the protocols we have described and not have to use buccal root torque as would be need with other techniques.

In comparing the open and the closed methods of bringing palatally impacted canine teeth into place it seems clear to us that the prognosis is better with the open method. With it, orthodontists are far more likely to achieve almost ideal results. Unfortunately this technique is more uncomfortable for patients, but it is more rapid.

\section{4 - CONCLUSIONS}

Both the methods we have discussed give good results.

Orthodontists will find the open method to be less rigorous and demanding and more conducive to precise control of the impacted tooth's movement.

By placing grafts of attached gingiva to buccally impacted upper canine teeth orthodontists will simplify treatment and reinforce the health of the
On the other hand we do not yet know if the results of one technique are more stable than the results of the other.

It is possible that the open traction procedure requires more retention time that the tunnelised technique because over its trajectory the canine will have traumatised palatal mucosa over a long distance while the tunnelised method will have brought the canine into its final position along a shorter trajectory. periodontium but unfortunately this procedure may increase the risk of relapse and worsen the aesthetic result.

In order to reduce the prominence of the root, orthodontists should first move palatally impacted canine teeth horizontally in a high position toward their buccal position and then give them a rest period before bringing them down into their final positions.

\section{BIBILIOGRAPHY}

1. Becker A, Casap N, Chaushu S. Conventional wisdom and the surgical exposure of impacted teeth. Orthod Craniofac Res 2009;12:82-93. Review.

2. Schmidt AD, Kokich VG, Periodontal response to early uncovering, autonomous eruption, and orthodontic alignment of palatally impacted maxillary canines. Am J Orthod Dentofacial Orthop 2007 Apr;131(4):449-55.

3. Vanarsdall RL, Corn H. Soft tissue management of labially positioned unerupted teeth. Am J Orthod 1977;72:53-64.

4. Vermette ME, Kokich VG, Kennedy DB. Uncovering labially impacted teeth: apically positioned flap and closed eruption technique. Angle Orthod 1994;65:23-32. 